\title{
Long term VHE $\gamma$-ray monitoring of bright blazars with a dedicated Cherenkov telescope
}

\author{
T. Bretz ${ }^{a}$, M. Backes ${ }^{b}$, D. Neise ${ }^{b}$, W. Rhode ${ }^{b}$, K. Mannheim ${ }^{a}$, D. Dorner ${ }^{*}, a$, \\ D. Hadasch ${ }^{b}$, J. K. Becker ${ }^{b}$, A. Biland ${ }^{c}$, I. Braun ${ }^{c}$, I. Britvitch ${ }^{c}$, S. C. Commichau ${ }^{c}$, \\ M. Rissi ${ }^{c}$, H. von Gunten ${ }^{c}$, D. Hildebrand ${ }^{c}$, D. Kranich ${ }^{c}$, E. Lorenz ${ }^{c}$, W. Lustermann ${ }^{c}$, \\ F. Pauss ${ }^{c}$, M. Pohl ${ }^{d}$, D. Renker ${ }^{e}$, U. Röser ${ }^{c}$, U. Straumann ${ }^{f}$, G. Viertel ${ }^{c}$ \\ ${ }^{a}$ Universität Würzburg, 97074 Würzburg, Germany \\ ${ }^{b}$ Technische Universität Dortmund, 44221 Dortmund, Germany \\ ${ }^{c}$ ETH Zürich, 8093 Zürich, Switzerland \\ ${ }^{d}$ University of Geneva, CH-1211 Geneva, Switzerland \\ ${ }^{e}$ Paul Scherrer Institut (PSI) Villingen, CH-5232 Villingen, Switzerland \\ ${ }^{f}$ Universität Zürich, CH-8057 Zürich, Switzerland \\ E-mail: tbretz@astro.uni-wuerzburg.de, \\ michael.backes@physik.tu-dortmund.de, isabel.braun@phys.ethz.ch, \\ dornercastro.uni-wuerzburg.de
}

\begin{abstract}
We intend to set up an imaging air Cherenkov telescope with low cost, but high performance design for remote operation. The goal is to dedicate this $\gamma$-ray telescope to long-term monitoring observations of nearby, bright blazars at very high energies (VHE). We will (i) search for orbital modulation of the blazar emission due to supermassive black hole binaries, (ii) study the statistics of flares and their physical origin, and (iii) correlate the data with observations of flares with higher sensitivity telescopes such as MAGIC, VERITAS, and H.E.S.S. Common observations with the Whipple $10 \mathrm{~m}$-monitoring telescope will be the first step towards a future $24 \mathrm{~h}$-monitoring of selected sources. This idea was presented for the first time in [1]. The telescope design is based on a full technological upgrade of one of the former telescopes of the HEGRA collaboration, still located at the Observatorio del Roque de los Muchachos on the Canarian Island of La Palma (Spain). After this upgrade, the telescope will be operated robotic, its sensitivity will greatly be improved and a much lower energy threshold below $350 \mathrm{GeV}$ will be achieved.
\end{abstract}

Workshop on Blazar Variability across the Electromagnetic Spectrum April 22-25 2008

Palaiseau, France

${ }^{*}$ Speaker. 


\section{Introduction}

Since the termination of the HEGRA observations, the succeeding experiments MAGIC [2] and H.E.S.S. [3], along with VERITAS [4] and CANGAROO [5], have impressively extended the physical scope of $\gamma$-ray observations, e.g. [6]. This became possible by lowering the energy threshold from $700 \mathrm{GeV}$ to less than $100 \mathrm{GeV}$ and increasing at the same time the sensitivity by a factor of five.

To fully exploit the discovery potential of the improved sensitivity, the discovery of new, faint objects has become the major task for the new telescopes. A diversity of astro-physical sources can be studied with these telescopes and therefore limits their availability for monitoring purposes of well-known bright sources. There are, however, strong reasons to make an effort for the continuous monitoring of the few exceptionally bright blazars. This can be achieved by operating a dedicated monitoring telescope of the HEGRA-type, referred to in the following as DWARF (Dedicated multiWavelength Agn Research Facility).

\section{Science case}

The variability of blazars, seen across the entire electromagnetic spectrum, arises from the dynamics of relativistic jets and the underlying particle acceleration mechanisms. The jets are launched in the vicinity of accreting supermassive black holes. Theoretical models predict variability arising from the interplay between jet expansion, particle injection, acceleration and cooling [7].

To overcome the limitations of biased sampling, a complete monitoring database for variability investigations of a few representative bright sources needs to be built up.

DWARF will run as a facility dedicated to long-term monitoring of few selected blazars only, providing a maximum observation time for the program. These sources will be: Mrk 421, Mrk 501, 1 ES 2344+514, 1 ES 1959+650, H 1426+428, PKS 2155-304. At least one of the proposed targets will be visible any time of the year.

In detail the following investigations are planned:

- As a direct result of the measurements, the duty cycle, the baseline emission, and the power spectrum of flux variations will be determined and compared with variability properties in other wavelength ranges.

- The lightcurves will be interpreted using models for the nonthermal emission from relativistically expanding plasma jets.

- The black hole mass and accretion rate will be determined from the emission models. Estimates of the black hole mass from emission models, a possible orbital modulation, and the Magorrian relation (relating the black hole mass with the stellar bulge mass of the host galaxy) [8] will be compared.

- When flaring states will be discovered during the monitor program, MAGIC will issue a Target of Opportunity observation to obtain better time resolution.

- Correlating the arrival times of neutrinos detected by the neutrino telescope IceCube with simultaneous measurements of DWARF will allow to test the hypothesis that flares in blazar jets are connected to hadronic emission processes and thus to neutrino emission from these 
sources $[9,10]$. The investigation proposed here is complete for both neutrino and $\gamma$-observations, and can therefore lead to conclusive results.

- The diffuse flux of escaping UHE cosmic rays obtained from AUGER or flux limits of neutrinos from IceCube, respectively, will be used to constrain models of UHE cosmic ray origin and large-scale magnetic fields.

- Multi-frequency observations together with the Metsähovi Radio Observatory and the optical Tuorla Observatory are planned. The measurements will be correlated with INTEGRAL and GLAST results, when available. X-ray monitoring using the SWIFT and Suzaku facilities will be proposed.

- The most ambitious scientific goal of this proposal is the search for signatures of binary black hole systems from orbital modulation of VHE $\gamma$-ray emission [11]. In case of a confirmation of the present hints in the temporal behaviour of Mrk 501 [12], gravitational wave templates could be computed with high accuracy to establish their discovery with LISA.

Furthermore, operating a smaller but robotic telescope is an essential contribution to the next plans in ground-based gammma ray astronomy. It is necessary to obtain know-how for the operation of future networks of Cherenkov telescopes, e.g. a monitoring array around the globe or a single-place array like CTA [13] or AGIS [14].

\section{Technical setup}
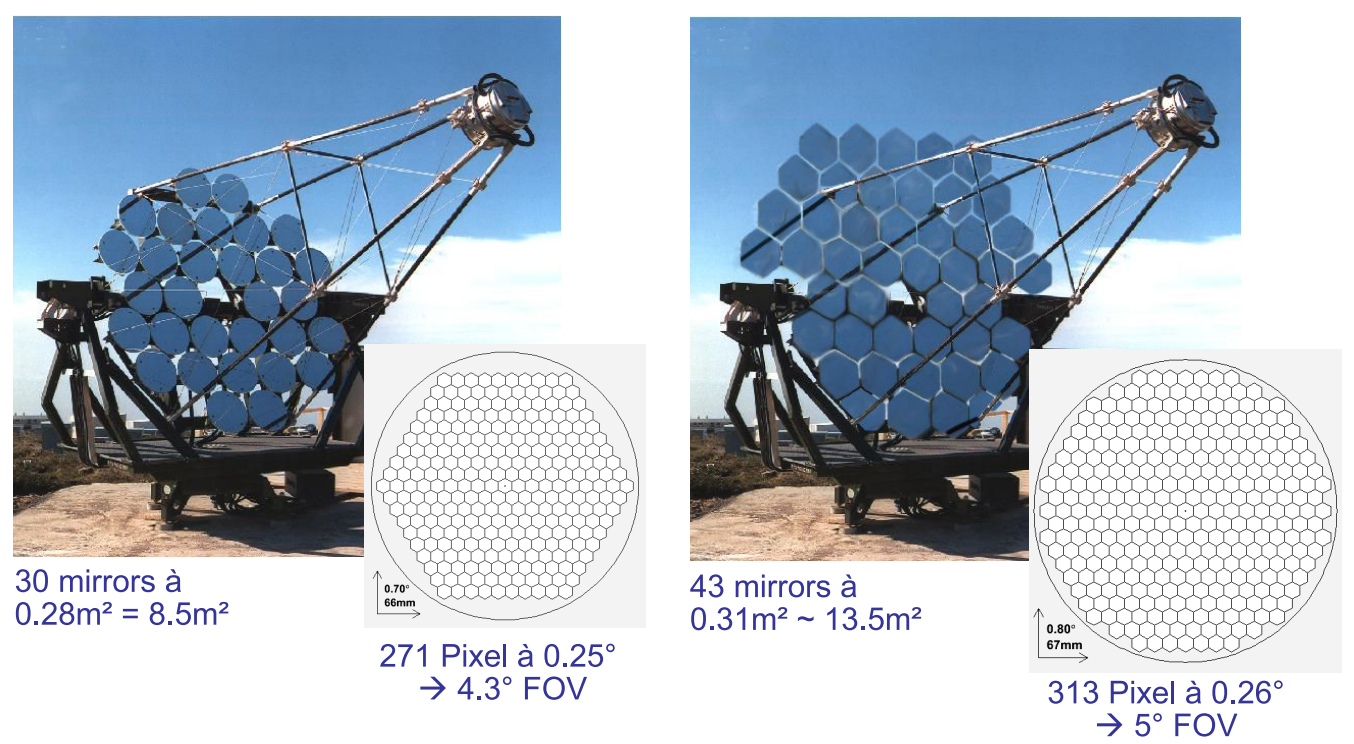

Figure 1: Left: picture of the HEGRA CT3 when it was still operational and a schematic view of the camera plane. Right: Photomontage of the DWARF telescope and a schematic view of the camera plane assuming a PMT camera setup.

Telescope structure The former HEGRA telescope CT3 at the Oberservatorio del Roque de los Muchachos (ORM), now owned by the MAGIC collaboration, will be refurbished and equipped for robotic operation to reduce costs and man power demands. 
Drive system The drive system should allow for relatively fast repositioning for three reasons:

- Fast movement is in most cases mandatory for future ToO observations.

- Wobble-mode observations will be done changing the wobble-position continuously (each $20 \mathrm{~min}$ ) for symmetry reasons.

- To ensure good time coverage of more than one source visible at the same time, the observed source will be changed in constant time intervals ( $\sim 20 \mathrm{~min})$.

Therefore three 150 Watt servo motors are intended. A microcontroller based motion control unit (SPS) similar to the one of the current MAGIC II drive system will be used. For communication with the readout-system a standard Ethernet connection based on the TCP/IP-protocol is applied.

Mirrors The existing mirrors are replaced by new plastic mirrors. The cheap and light-weight material has formerly been used for Winston cones flown in balloon experiments. The mirrors are copied from a master and coated with a reflecting and a protective material. By a change of the mirror geometry the mirror area can be increased from $8.5 \mathrm{~m}^{2}$ to $13 \mathrm{~m}^{2}$; this includes an increase of $\sim 10 \%$ per mirror by using a hexagonal layout (see Fig. 1).

Pointing calibration To correct for misalignments of the telescope axis a pointing correction algorithm as used in the MAGIC tracking system will be applied. It is calibrated by measuring the reflection of bright guide stars on the camera surface and ensures a pointing accuracy well below the pixel diameter. Therefore a high sensitive low-cost video camera, as already in operation for MAGIC I and II, will be installed.

Camera For long-term observations a good background estimation, which keeps the systematic errors small, is mandatory. The best choice for this is the synchronous background determination from the night-sky observed in the same field-of-view (FoV) with the same instrument (so called wobble-mode). This observation mode increases the sensitivity by at least a factor of $\sqrt{2}$ as dedicated background observations become obsolete, which also ensures a better time coverage of the observed sources. Having a camera with a FoV of about $3.5^{\circ}-5^{\circ}$ allows more than one independent position for background estimation and by this increases sensitivity further by better background statistics. To decrease the dependence of the measurement on the camera geometry, a round camera layout will be chosen.

One possible solution is a classical 313 pixel PMT camera based on the experience with HEGRA and MAGIC. Photomultipliers with a diameter of $19 \mathrm{~mm}$ and a quantum efficiency improved by $20 \%$ with respect to the CT3 system are considered. They ensure a granularity sufficient to guarantee good results even below the flux peak energy.

The preferred option is a novel camera based on Geigermode Avalanche Photo Diodes (G-APDs, also known as SiPMs or MPPCs) that is currently developed by a collaboration of Swiss institutes (ETH \& University Zurich, PSI, University of Geneva, EPF Lausanne). G-APDs promise a highly enhanced photon detection efficiency compared to PMTs, which lowers the achievable energy threshold for a given mirror area. In addition, they have a very reproducible output signal per photoelectron and practical advantages including a lower bias voltage and insensitivity to accidental exposure to light. Tests leading to the first detection of Cherenkov light from air showers are presented in [15]. 

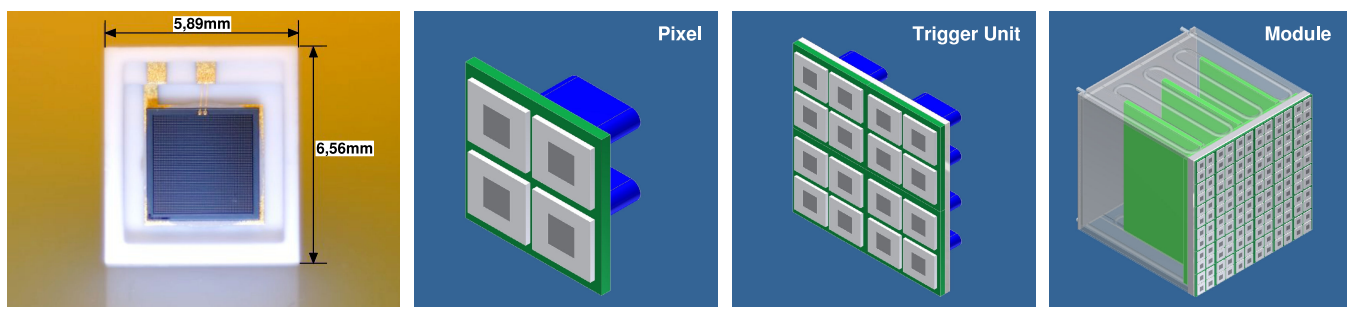

Figure 2: From left to right: the Hamamatsu G-APD (MPPC S10362-33-100C), layout of a pixel $(2 \times 2$ GAPDs), a unit (16 G-APDs) and a module composed of $6 \times 6$ pixels (144 G-APDs).

The G-APD selected for the camera project (Hamamatsu MPPC S10362-33-100C [16]) has a dimension of $3 \times 3 \mathrm{~mm}^{2}$ and consists of 900 cells of $100 \times 100 \mu \mathrm{m}^{2}$ size. To reduce the number of readout channels, four G-APDs will be added linearly forming a "pixel" (see Fig. 2). The installation will be done in three stages: a test module (Module 0) with $1^{\circ}$ field of view, an intermediate camera of $3^{\circ}$ diameter (Module 1) that will be tested on the $\mathrm{Crab}$ nebula $\mathrm{TeV}$ source, and the final $5^{\circ}$ camera (Module 2) for regular observations. For further details see [17].

Camera support For both camera solutions a further improvement of the quantum efficiency of about $8 \%$ can be achieved by applying a special wavelength shifting coating on the photon detectors. By over-coating the camera window with an anti-reflex layer of magnesium-fluoride a gain in transmission of 5\% is expected. Each individual G-APD or PM will be equipped with a specially designed light collector (modified Winston cone) to increase the light collection area. Their light-collection efficiency will be improved by using a highly reflecting mirror-foil, reaching a reflectivity in the order of $98 \%$. In total this will gain another $\sim 15 \%$ in light-collection efficiency compared to the CT3 system. Solid glass cones utilizing total reflectance are also considered as lights guides for the G-APD camera.

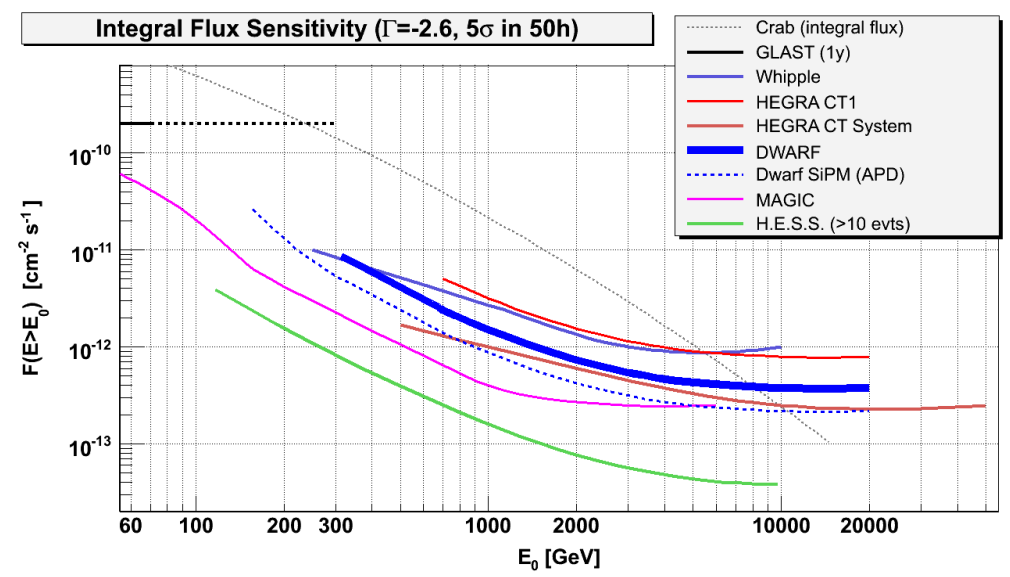

Figure 3: Integral flux sensitivity of current and former Cherenkov telescopes as well as the expectations for the dedicated long-term monitoring telescope DWARF, both with a PMT and a G-APD camera $[2,18,19]$. 
Data acquisition For the data acquisition system a hardware readout based on an analog sampling chip (Domino II/IV, developed at PSI [20]), currently designed for the MAGIC II readout [21], will be used. The low power consumption will allow to include the digitization near the signal source which makes an analog signal transfer obsolete. The advantage is less pick-up noise and less signal dispersion. By high sampling rates ( $2 \mathrm{GHz}$ or more), the over-all sensitivity will further be increased, because the short integration time allows for almost perfect suppression of noise due to night-sky background photons [22]. Assuming conservatively storage of raw-data at a readout rate of $30 \mathrm{~Hz}$ the storage space needed is less than $420 \mathrm{~GB} /$ month or $5 \mathrm{~TB} / y e a r$. The available automatic analysis package developed for MAGIC is modular and flexible, and can thus be used with minor changes for the DWARF project [23].

\section{Future extensions}

The known duty cycle of $\sim 1000 \mathrm{~h} /$ year for a Cherenkov telescope operated at La Palma limits the time-coverage of the observations. Therefore we propose a worldwide network of $(<10)$ small scale Cherenkov telescopes to be build in the future: A system so far unique in this energy range, which will allow $24 \mathrm{~h}$ monitoring of the bright AGN. The approved cooperation with the Whipple $10 \mathrm{~m}$-telescope is the first step in this direction.

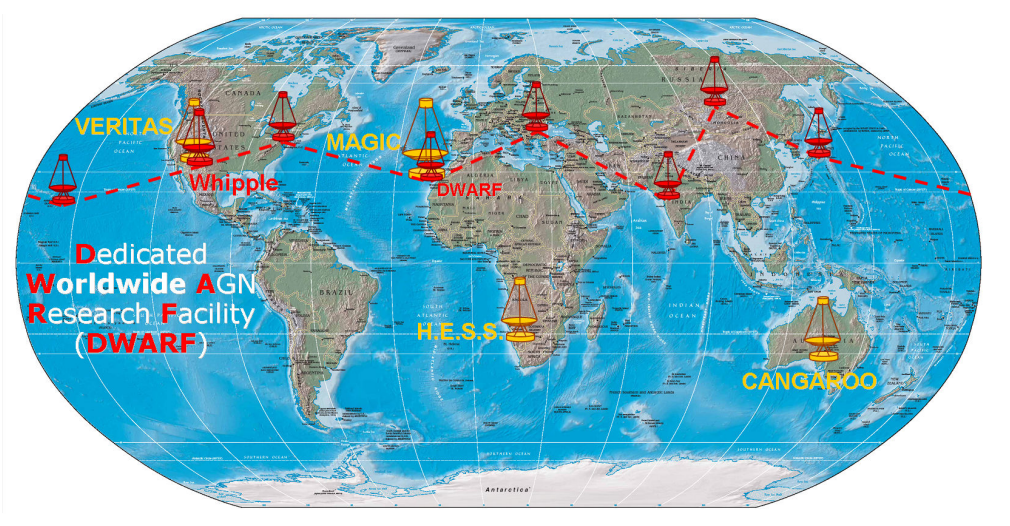

Figure 4: Possible distribution of Cherenkov telescopes in a future worldwide network for AGN monitoring.

\section{Conclusion}

The setup of a small telescope dedicated for long-term AGN monitoring is easily feasible. Such an activity is motivated by a variety of physical questions to be answered by the integration of this instrument in multiwavelength observations.

\section{Acknowledgments}

We would like to thank Riccardo Paoletti, Maria Victoria Fonseca and José Luis Contreras for intense discussions and Christian Spiering, Trevor Weekes, Leo Takalo, Merja Tornikolski, Brenda Dingus, Maria Magdalena Gonzalez Sanchez and the MAGIC collaboration for helpful support. 


\section{References}

[1] T. Bretz et al., Long-term VHE $\gamma$-ray monitoring of bright blazars with a dedicated Cherenkov telescope, in proceedings of the $30^{\text {th }}$ International Cosmic Ray Conference, 2007, to be published

[2] MAGIC collaboration, official homepage, http://magi c . mppmu.mpg . de, 2008

[3] H.E.S.S. collaboration, official homepage, http : / / www . mpi-hd.mpg . de/hfm/HES S, 2008

[4] VERITAS collaboration, official homepage, http://veritas.sao.arizona.edu, 2008

[5] CANGAROO team, official homepage, http: / / i crhp9. icrr.u-tokyo.ac.jp, 2008

[6] M. Teshima, Gamma Astronomy Summary, Presentation at TeV Particle Astrophysics II, available at http://www. icecube.wisc.edu/tev/, 2006

[7] M. Böttcher, Broadband continuum variability of blazars: General results and application to BL Lacertae in 2000, American Astronomical Society Meeting 35 (724), 2003

[8] J. Magorrian et al., The Demography of Massive Dark Objects in Galaxy Centers, The Astronomical Journal, 115 (2285), 1998

[9] A. Mücke, R. J. Protheroe, R. Engel, J. P. Rachen \& T. Stanev, BL Lac objects in the synchrotron proton blazar model, Astroparticle Physics, 18 (593), 2003

[10] K. Mannheim, The proton blazar, Astronomy \& Astrophysics, 269 (67), 1993

[11] F. M. Rieger \& K. Mannheim, Astronomy \& Astrophysics, 359 (948), 2000

[12] F. M. Rieger \& K. Mannheim, A possible Black Hole Binary in Mkn 501, in American Institute of Physics Conference Series 558 (716), 2001

[13] CTA collaboration, official homepage, http: / / www . cta-observatory • org, 2008

[14] AGIS collaboration, official homepage, http://gamma1.astro.ucla.edu/agis, 2008

[15] A. Biland et al., Nuclear Instruments and Methods in Physics Research A, 581 (143), 2007

[16] MPPC data sheet of Hamamatsu Photonics:

http://sales.hamamatsu.com/assets/pdf/catsandguides/mppc_kapd0002e03.pdf

[17] I. Braun et al., First Avalanche-diode Camera Test (FACT): A novel Camera using a G-APD Array for the Observation of very high-energy $\gamma$-Rays with Cherenkov Telescopes, presented at $5^{\text {th }}$ International Conference on New Developments In Photodetection 2008, Aix-les-Bains, to be published in Nuclear Instruments and Methods in Physics Research A

[18] N. Magnussen et al., The MAGIC Telescope Project for Gamma Astronomy above $10 \mathrm{GeV}$, in proceedings of $36^{\text {th }}$ INFN ELOISATRON Workshop on New Detectors, 1997 [astro-ph/9805.184]

[19] V. Vassiliev et al., in proceedings of the $26^{\text {th }}$ International Cosmic Ray Conference, 299, 1999

[20] S. Ritt, Design and Performance of the 5 GHz Waveform Digitizing Chip DRS3, Nucl. Science Symposium Conference Report 4 (2007) 2485-2488, NSS Õ07, IEEE.

[21] R. Pegna et al., A GHz sampling DAQ system for the MAGIC-II telescope, Nuclear Instruments and Methods in Physics Research A, 552 (382), 2007

[22] D. Tescaro et al., Study of the performance and capability of the new ultra-fast 2 GSample/s FADC data acquisition system of the MAGIC telescope, in proceedings of the $30^{\text {th }}$ International Cosmic Ray Conference, 2007, to be published

[23] T. Bretz et al., in proceedings of the $29^{\text {th }}$ International Cosmic Ray Conference, 315, 2005 